\title{
The Effects of Bitter Melon (Momordica charantia) Extracts on Serum and Liver Lipid Parameters in Hamsters Fed Cholesterol-Free and Cholesterol-Enriched Diets
}

\author{
Gamarallage V. K. SENANAYAKE ${ }^{1}$, Mitsuru MARUYAMA ${ }^{1}$, Masanobu SAKONO ${ }^{1}$, \\ Nobuhiro FUKUdA ${ }^{1, *}$, Toshiro Morishita ${ }^{2}$, Chizuko YUKIZAKI ${ }^{2}$, Mikio KAWANO ${ }^{2}$ \\ and Hideaki OHTA ${ }^{3}$ \\ ${ }^{1}$ Department of Biochemistry and Applied Biosciences, Faculty of Agriculture, University of Miyazaki, \\ Miyazaki 889-2192, Japan \\ ${ }^{2}$ Miyazaki Prefectural Food Development Center, Miyazaki 880-0303, Japan \\ ${ }^{3}$ Faculty of Nutritional Science, Nakamura Gakuen University, Fukuoka 814-0198, Japan
}

(Received November 12, 2003)

\begin{abstract}
Summary The hypolipidemic effect of dietary methanol fraction (BMMF) extracted from bitter melon (Koimidori variety), at the levels of $0.5 \%$ and $1.0 \%$, was examined in male golden Syrian hamsters fed diets supplemented with and without cholesterol. The feeding of BMMF at $0.5 \%$ and $1.0 \%$ levels in the diets for $4 \mathrm{wk}$ tended to reduce food intake and growth, although there was no difference in food efficiency (weight gain/food intake). An effect of dietary BMMF on serum triglyceride was not seen in hamsters fed diets free of cholesterol, while hypertriglyceridemia induced by dietary cholesterol was significantly lowered in a dose-dependent manner in those fed diets containing the BMMF. Serum total cholesterol concentration also tended to decrease in a dose-dependent manner following feeding of increasing amounts of BMMF in the presence and absence of cholesterol in the diet. The effects of dietary BMMF on liver triglyceride and total cholesterol levels were marginal, although dietary cholesterol caused a marked accumulation of these lipid molecules in the liver. These results suggest that the BMMF contains some components that could ameliorate lipid disorders such as hyperlipidemia.
\end{abstract}

Key Words bitter melon, hamster, serum, liver, lipid profiles

Metabolic and physiological effects of certain vegetables and herbs have garnered considerable interest in the past due to their perceived health benefits, especially in their ability to prevent life style-related diseases such as hyperglycemia, fatty liver, hyperlipidemia and coronary heart disease (1).

Bitter melon (Momordica charantia) is a vegetable cultivated in the tropical or sub-tropical regions of South America and Asia. It has been used as a traditional remedy for various illnesses, particularly diabetes mellitus $(2,3)$. Clinical investigations in humans have also reported beneficial effects of bitter melon in preventing and/or relieving hyperglycemia in human type II diabetes $(4,5)$. Animal experiments have further substantiated these claims, and it has been shown there are hypoglycemic effects in alloxan- or streptozotocininduced diabetic animals (6-9). However, research on bitter melon has mainly focused on its purported antidiabetic properties, in spite of the possibility that bitter melon might affect lipid metabolism as well, due to the interconnection between carbohydrate and lipid metabolism.

In previous experiments, we reported for the first

\footnotetext{
* Corresponding author.

E-mail: fukudan@cc.miyazaki-u.ac.jp
}

time that the dietary Olympia variety of bitter melon, as a freeze-dried powder, has a lowering effect on hepatic triglyceride levels, in addition to its well-known hypoglycemic effect, in the rat (10). We have further confirmed that this activity is highest in an indigenous variety of melon named Koimidori, followed by two other varieties, Hyakunari and Powerful-Reishi. We further carried out fractionation of Koimidori powder with organic solvents such as hexane, acetone and methanol to isolate the active component(s), and found that it was localized in the fraction extracted by methanol (BMMF) (11).

On the other hand, it was recently reported that longterm feeding of bitter melon juice resulted in a significant reduction in the concentration of serum lipids in the streptozotocin-induced hyperlipidemic rats, but only total cholesterol was reduced in the non-treated normal rats fed bitter melon juice (12). Chen et al. (13) recently reported that feeding of freeze-dried bitter melon juice resulted in improved insulin resistance and lower visceral adipose tissue weight, serum insulin and leptin, but that it raised serum-free fatty acid concentrations in rats fed a high-fat diet. These observations suggest that the edible portion of bitter melon contains some components that influence lipid metabolism, probably through hormonal regulation. 
In the present study, we examined the lipid-lowering activity of BMMF in male golden hamsters fed a diet with or without $0.2 \%$ cholesterol supplementation. Hamsters are a useful model system for studying lipid metabolism as they share many similarities with humans in lipid metabolism and in cholesterol and atherogenic lipoprotein cholesterol (LDL and VLDL) responses to atherogenic diets (14). Furthermore, the hypolipidemic activity of bitter melon extract has not been extensively investigated in hamsters.

\section{MATERIALS AND METHODS}

The methanol fraction of the Koimidori variety of bitter melon, which was developed and cultivated at the Miyazaki Agricultural Experimental Station (Miyazaki, Japan), was prepared according to a method reported elsewhere $(10)$. In brief, freeze-dried powder of bitter melon was mixed with $10 \mathrm{vol}$ of $n$-hexane for $1 \mathrm{~h}$ with continuous stirring at room temperature, and was followed by centrifugation. The supernatant was discarded by decantation; the resulting precipitate was mixed again with $10 \mathrm{vol}$ of $n$-hexane for another $1 \mathrm{~h}$ of continuous stirring. After centrifugation, the supernatant was again discarded by decantation and the resulting precipitate was subjected successively to stirring in $10 \mathrm{vol}$ of acetone for $1 \mathrm{~h}$ and centrifugation, and the supernatant was discarded. The resulting precipitate was extracted with methanol; this was done twice following the same procedure described above. The combined supernatant was condensed to dryness using a rotary evaporator in vacuo, and was kept under a fume hood until the odor of organic solvents had dissipated. It was then used for dietary test materials as the methanol fraction (BMMF). BMMF component(s) was identified qualitatively by Libermann-Burchard reaction, by its hemolytic activity toward rat erythrocytes, and by its precipitability by $\mathrm{Ba}(\mathrm{OH})_{2}$, according to the method of $\mathrm{Ng}$ et al. (15). In brief, the unsaponifiable matters of BMMF were dissolved in acetic anhydrate, followed by the addition of concentrated sulfuric acid, which revealed the appearance of a bright coloration. In the hemolytic analysis of rat erythrocytes, suspended in phosphate-buffered saline $(\mathrm{pH} 7.4)$ at 2\%, BMMF caused a potent hemolytic activity. Further, in the $\mathrm{Ba}(\mathrm{OH})_{2}$ precipitation test, the presence of saponins was confirmed by precipitation formation upon the addition of a saturated solution of $\mathrm{Ba}(\mathrm{OH})_{2}$. Thus, these all qualitative analyses revealed the presence of saponins in the BMMF, although more detailed analyses of BMMF were carried out in our laboratory. On the other hand, gas liquid chromatographic analysis (16) of BMMF revealed no presence of plant sterols.

Male golden Syrian hamsters (weighing 77-97 g), purchased from a local breeder (Nihon SLC Co., Shizuoka, Japan), were given a powdered commercial diet (Type CE-2, Clea Japan, Tokyo, Japan) ad libitum and water for several days in a room at constant temperature $\left(22-24^{\circ} \mathrm{C}\right)$ with a 12 -h light-dark cycle (light on 07:00-19:00). They were then divided into six groups with 6-7 animals in each group. The cholesterol-free diet was prepared according to the recommendations of the American Institute of Nutrition (17) and contained (\% by weight): casein, 20; lard, 9; corn oil, 1; vitamin mixture (AIN 76), 1.0; mineral mixture (AIN 76), 3.5; DL-methionine, 0.3; choline bitartrate, 0.2; cellulose, 5; cornstarch, 15; and sucrose to $100 \%$. The cholesterol diet was prepared by adding $0.2 \%$ cholesterol to the cholesterol-free diet at the expense of sucrose. BMMF was added at the levels of $0.5 \%$ and $1 \%$ to respective cholesterol-free and cholesterol-enriched diets at the expense of sucrose. Hamsters had free access to the diets and water for $4 \mathrm{wk}$, with food consumption and body weight being recorded every other day.

Each animal was anesthetized with an intraperitoneal injection of pentobarbital sodium and blood was withdrawn from posterior vena cava. The liver was removed immediately after the animal was sacrificed, rinsed with ice-cold saline, dried on filter paper, and weighed following the removal of extra-hepatic tissues. The experimental protocol was approved by the Ethical Committee for Animal Experiments of the Faculty of Agriculture, University of Miyazaki, Miyazaki, Japan.

The serum and liver were stored at $-80^{\circ} \mathrm{C}$ until analyses were done. Serum triglyceride, total-, free- and HDL-cholesterol, and phospholipids were determined enzymatically using available commercial kits: Triglycerides-E Test, Total cholesterol-C Test and Phospholipids-B Test were from Wako Chemicals (Osaka, Japan), and HDL-cholesterol from Daiichi Pure Chemicals Co. Ltd. (Tokyo, Japan). Liver lipids were extracted and purified according to Folch et al. (18), and triglyceride, cholesterol and phospholipids were measured chemically as described elsewhere (19). Cholesterol ester was estimated as the difference between total and free cholesterol. The atherogenic index was calculated by the following formula: (Total cholesterol-HDL-cholesterol)/ HDL-cholesterol.

Statistics. Data were analyzed by one-way analysis of variance, followed by multiple comparisons with Turkey-Kramers test (StatView software, Sas Institute, USA), and the statistical significance of the difference of the means was evaluated at the level of $p<0.05$.

\section{RESULTS}

\section{Food intake, growth, and liver weight (Table 1)}

Feeding of the methanol fraction (BMMF) extracted from the Koimidori variety of bitter melon at $0.5 \%$ and $1 \%$ levels caused a tendency toward reduction in food intake and body weight gain as compared to the control hamsters fed diets free of BMMF, both in the presence and absence of dietary cholesterol. However, there was no significant difference in food efficiency (weight gain/ food intake) among the various groups. Hamsters fed diets containing cholesterol had higher liver weight as compared to the animals fed diets free of cholesterol. The addition of BMMF to the cholesterol-enriched diet tended to prevent liver enlargement, although no statistical significance was noted.

Serum and liver lipids (Table 2)

The addition of cholesterol to the cholesterol-free diet 
Table 1. Effect of methanol fraction of bitter melon on growth parameters in hamsters fed cholesterol-free and cholesterolenriched diets.*

\begin{tabular}{|c|c|c|c|c|c|c|}
\hline & \multicolumn{3}{|c|}{ Cholesterol-free } & \multicolumn{3}{|c|}{ Cholesterol-enriched } \\
\hline & Control & $0.5 \% \mathrm{BMMF}$ & $1 \% \mathrm{BMMF}$ & Control & $0.5 \%$ BMMF & $1 \% \mathrm{BMMF}$ \\
\hline \multicolumn{7}{|l|}{ Growth parameters } \\
\hline Initial body weight $(\mathrm{g})$ & $86.8 \pm 2.2$ & $86.6 \pm 2.2$ & $86.9 \pm 1.7$ & $86.4 \pm 2.3$ & $84.7 \pm 1.8$ & $86.9 \pm 1.7$ \\
\hline Final body weight $(\mathrm{g})$ & $114 \pm 4$ & $109 \pm 2$ & $101 \pm 4$ & $114 \pm 5$ & $104 \pm 2$ & $102 \pm 5$ \\
\hline Food intake $(\mathrm{g} / \mathrm{d})$ & $9.83 \pm 0.37^{\mathrm{ab}}$ & $8.37 \pm 0.29^{\mathrm{a}}$ & $8.18 \pm 0.45^{\mathrm{a}}$ & $10.5 \pm 0.5^{b}$ & $8.77 \pm 0.64^{\mathrm{ab}}$ & $8.74 \pm 0.41^{\mathrm{ab}}$ \\
\hline Food efficiency ( $\mathrm{g} / \mathrm{kg})$ & $98.0 \pm 12.2$ & $98.5 \pm 6.8$ & $83.2 \pm 16.6$ & $86.0 \pm 13.5$ & $87.7 \pm 9.4$ & $83.0 \pm 14.6$ \\
\hline Liver weight (g/100 g BW) & $5.01 \pm 0.20^{\mathrm{abc}}$ & $4.91 \pm 0.11^{\mathrm{ac}}$ & $4.71 \pm 0.10^{\mathrm{a}}$ & $5.59 \pm 0.23^{b c}$ & $5.63 \pm 0.09^{b}$ & $5.39 \pm 0.22^{\mathrm{abc}}$ \\
\hline
\end{tabular}

* The values are means \pm SE for seven to eight hamsters per group. ${ }^{\text {abc }}$ Values not sharing common superscript letters in the same row are significantly different at $p<0.05$. BMMF, methanol fraction of bitter melon.

Table 2. Effect of methanol fraction of bitter melon on serum and liver lipid parameters in hamsters fed cholesterol-free and cholesterol-enriched diets.*

\begin{tabular}{|c|c|c|c|c|c|c|}
\hline & \multicolumn{3}{|c|}{ Cholesterol-free } & \multicolumn{3}{|c|}{ Cholesterol-enriched } \\
\hline & Control & $0.5 \% \mathrm{BMMF}$ & $1 \% \mathrm{BMMF}$ & Control & $0.5 \% \mathrm{BMMF}$ & $1 \% \mathrm{BMMF}$ \\
\hline \multicolumn{7}{|c|}{ Serum parameters $(\mathrm{mg} / \mathrm{dL})$} \\
\hline Triglyceride & $263 \pm 19^{a}$ & $288 \pm 24^{\mathrm{a}}$ & $308 \pm 37^{a}$ & $464 \pm 47^{b}$ & $343 \pm 14^{\mathrm{ab}}$ & $296 \pm 20^{a}$ \\
\hline \multicolumn{7}{|l|}{ Cholesterol } \\
\hline Total & $234 \pm 7^{\mathrm{ab}}$ & $186 \pm 22^{\mathrm{a}}$ & $187 \pm 9^{a}$ & $276 \pm 21^{b}$ & $222 \pm 21^{\mathrm{ab}}$ & $193 \pm 12^{\mathrm{ab}}$ \\
\hline HDL & $93.8 \pm 2.2^{\mathrm{ab}}$ & $82.4 \pm 7.2^{\mathrm{a}}$ & $79.9 \pm 3.0^{\mathrm{a}}$ & $108 \pm 7^{\mathrm{b}}$ & $95.3 \pm 7.8^{\mathrm{ab}}$ & $82.7 \pm 4.0^{\mathrm{a}}$ \\
\hline Atherogenic index & $1.49 \pm 0.01^{\mathrm{ab}}$ & $1.35 \pm 0.06^{\mathrm{a}}$ & $1.37 \pm 0.03^{\mathrm{a}}$ & $1.55 \pm 0.03^{\mathrm{b}}$ & $1.45 \pm 0.05^{\mathrm{ab}}$ & $1.39 \pm 0.03^{\mathrm{ab}}$ \\
\hline Phospholipid & $222 \pm 21$ & $172 \pm 18$ & $166 \pm 8$ & $226 \pm 18$ & $205 \pm 20$ & $173 \pm 10$ \\
\hline \multicolumn{7}{|c|}{ Liver parameters (mg/g liver) } \\
\hline Triglyceride & $5.46 \pm 0.40^{\mathrm{a}}$ & $5.68 \pm 0.29^{\mathrm{ab}}$ & $5.87 \pm 0.21^{\mathrm{ab}}$ & $7.95 \pm 0.30^{c}$ & $7.26 \pm 0.32^{c}$ & $6.81 \pm 0.28^{b c}$ \\
\hline \multicolumn{7}{|l|}{ Cholesterol } \\
\hline Total & $6.11 \pm 0.47^{\mathrm{a}}$ & $5.38 \pm 0.34^{\mathrm{a}}$ & $4.37 \pm 0.25^{\mathrm{a}}$ & $34.7 \pm 2.2^{\mathrm{b}}$ & $30.3 \pm 1.6^{\mathrm{b}}$ & $33.7 \pm 1.1^{b}$ \\
\hline Free & $2.06 \pm 0.09^{\mathrm{a}}$ & $1.91 \pm 0.12^{\mathrm{a}}$ & $2.10 \pm 0.05^{\mathrm{a}}$ & $2.96 \pm 0.18^{b}$ & $2.44 \pm 0.14^{\mathrm{ab}}$ & $2.30 \pm 0.17^{\mathrm{a}}$ \\
\hline Ester (\%) & $65.5 \pm 2.3^{b}$ & $63.6 \pm 3.6^{\mathrm{b}}$ & $50.9 \pm 3^{a}$ & $91.4 \pm 0.5^{c}$ & $91.8 \pm 0.6^{c}$ & $93.1 \pm 0.7^{\mathrm{c}}$ \\
\hline Phospholipid & $24.5 \pm 0.9$ & $26.8 \pm 0.4$ & $26.1 \pm 0.4$ & $27.1 \pm 1.0$ & $26.0 \pm 0.4$ & $27.0 \pm 0.7$ \\
\hline
\end{tabular}

${ }^{*}$ The values are means \pm SE for seven to eight hamsters per group. ${ }^{\text {abc }}$ Values not sharing common superscript letters in the same row are significantly different at $p<0.05$. BMMF, Methanol fraction of bitter melon.

caused a significant increase in the concentration of serum triglyceride, while BMMF supplementation to the cholesterol-enriched diet, but not to the cholesterol-free diet, caused a dose-dependent reduction in the concentration of this lipid molecule, the extents of reduction being $26.1 \%$ and $36.2 \%$ in the hamsters fed diets containing BMMF at the levels of $0.5 \%$ and $1.0 \%$, respectively. Although the cholesterol-enriched diet did not significantly elevate the serum total cholesterol levels in the hamsters as compared to those fed diets that were not enriched, the supplementation of BMMF to the cholesterol-enriched diet caused $19.6 \%$ and $30.1 \%$ reductions in the concentration of serum total cholesterol, although the reduction in the concentration was not statistically significant at both $0.5 \%$ and $1 \%$ levels of dietary BMMF.

Cholesterol concentrations in the HDL fraction responded in the same manner as that observed for total cholesterol; the concentration tended to elevate following the feeding of cholesterol, while it was significantly lowered by concurrent supplementation of BMMF 1\% to the cholesterol-enriched diets. Atherogenic index (total cholesterol-HDL-cholesterol/HDL-cholesterol) tended to decrease in the BMMF groups as compared to the control groups in the presence and absence of dietary cholesterol, although the reduction was statistically insignificant.

Cholesterol feeding led to a 1.5 -fold increase in the concentration of hepatic triglyceride. BMMF tended to reduce liver triglyceride concentrations in the hamsters fed diets enriched with cholesterol, but not in those fed diets free of cholesterol. Cholesterol supplementation to the cholesterol-free diet caused a marked, 5.7-fold, accumulation of total cholesterol in the liver, while the free cholesterol concentration had only a small elevation, suggesting that the accumulation of total cholesterol due to dietary cholesterol was largely due to an increased cholesterol ester. Dietary BMMF tended to decrease the concentration of hepatic total cholesterol in a dose-dependent manner in the absence of dietary 
cholesterol, while free cholesterol levels remained unchanged; as a result, the cholesterol ester ratio was significantly lower following the feeding of BMMF $1 \%$. On the other hand, hamsters fed cholesterol-enriched diets supplemented with $1 \%$ BMMF, when compared to those fed the BMMF-free diet, caused no altered liver total cholesterol concentration, but they had significantly reduced concentrations of free cholesterol. Thus, BMMF had a small, but some effect on cholesterol metabolism in the hamster.

Hepatic phospholipid levels tended to increase following the feeding of BMMF in the absence of cholesterol, while those levels remained unchanged after feeding BMMF in the presence of dietary cholesterol.

\section{DISCUSSION}

Feeding BMMF at 0.5 and $1.0 \%$ levels tended to decrease the food intake in hamsters, leading to a somewhat decreased body weight gain when compared to the controls. It is known that food restriction or reduced food intake is, in some cases, associated with altered lipid metabolism (20). Thus, we cannot rule out the possibility that the tendency toward reduction in food intake and growth may have led to a decreased concentration of serum and liver lipids. However, food conversion efficiency, expressed as weight gain/food intake, was comparable among the various groups. On the other hand, we observed no reduction in the food intake and growth of rats fed diets containing 1\% BMMF as compared to those fed diets with no added BMMF, but a potent liver triglyceride-lowering effect was exhibited (11). Thus, the moderate suppressed food intake and growth caused by dietary BMMF are specific in hamsters, but not other experimental animals such as rats. These observations therefore suggest that the following altered concentration of serum and liver lipids induced by dietary BMMF could be attributed to the influence of dietary BMMF per se, rather than the suppressed food intake and growth.

We have previously reported that, in male rats fed for $14 \mathrm{~d}$ with a freeze-dried powder of bitter melon (Olympia variety), there was a marked reduction in the concentration of hepatic triglyceride and total cholesterol, while the effects on serum triglyceride and total cholesterol were marginal (10). Furthermore, we have found in another series of experiments using male rats, that the fraction extracted by methanol (BMMF) from an indigenous variety named Koimidori contains active component(s) capable of lowering liver triglyceride and cholesterol concentrations (11).

In this experiment, we examined the effect of BMMF on serum and liver lipid concentrations in the hamster. The hamster is a useful model for studying lipid metabolism, since the serum lipid concentration in hamsters fed a diet free of cholesterol is considerably higher than the concentration in rats fed the same diet (21). Furthermore, the supplementation of modest amounts of dietary cholesterol to a cholesterol-free for hamsters has been shown to cause a marked elevation of serum and liver lipids, which is consistent with present obser- vations. In contrast, in most other animal species, especially in rats, substantial amounts of cholesterol and bile acids must be added to a cholesterol-free diet to raise the serum lipid levels (10). These observations suggest that the hamster is well suited for the study of dietary ingredients that alter endogenous and exogenous hyperlipidemia.

The present study demonstrated a potent serum triglyceride-lowering activity of BMMF in the hamsters fed diets with cholesterol in a dose-dependent manner, but not in those fed diets with no added cholesterol. In addition, the elevated liver triglyceride concentration caused by dietary cholesterol tended to be lower in the hamsters fed with BMMF in a dose-dependent manner. These results suggest that dietary BMMF is effective in lowering serum and liver triglyceride in the hamster, especially with hypertriglyceridemia and fatty livers caused by dietary cholesterol. On the other hand, BMMF feeding caused a tendency toward reduction in the concentration of serum cholesterol in a dose-dependent manner in the hamsters fed diets, especially with cholesterol supplements.

The mechanism(s) responsible for the observed reduction in the concentration of serum lipids by dietary BMMF remains to be determined. However, bitter melon has been reported to contain some active components, namely saponin and plant sterols, to influence glucose and lipid metabolism (22). Some of these compounds are shown to have an inhibitory effect on lipid biosynthesis (15). We therefore preliminarily qualitatively identified the saponins by the methods described by $\mathrm{Ng}$ et al. (15) and plant sterols by gas liquid chromatography (16), and found the presence of saponins but not of plant sterols. It is known that saponins derived from other plant sources are effective in lowering liver triglyceride levels in experimental animals such as rats (23). These compounds have been shown to exhibit hypotriglyceridemic activity through probable inhibition of triglyceride synthesis in the liver (23); a similar mechanism might be the cause of the triglyceride lowering effect of BMMF demonstrated in this experiment, although clarification of these issues awaits further studies, after the chemical isolation of saponin from BMMF.

The results of this study, together with those of preceding experiments with rats, indicate a potential usefulness of BMMF for treating mild lipia disorders such as hyperlipidemia and fatty liver. Studies are in progress to identify the compound responsible for the observed efficacy, and to address the serum lipid-lowering mechanism using a radiolabeled acetate compound.

\section{Acknowledgments}

G.V.K. Senanayake is much obliged to the Japanese Government and University of Miyazaki for the provision of a scholarship grant from the Ministry of Education, Sports, Culture, Science and Technology. The authors express our thanks to the Japan Science and Technology Agency (JST) for the provision of research funds. The authors also wish to thank Dr. Udaya de 
Silva of the Animal Science Department, Oklahoma State University, USA, for his valuable comments in the preparation of this manuscript.

\section{REFERENCES}

1) Ferrari CKB, Torres EAFS. 2003. Biochemical pharmacology of functional foods and prevention of chronic diseases of aging. Biomed Pharmacother 57: 251-260.

2) Karunanayake EH, Welihinda J, Sirimanne SR, Sinnadorai G. 1984. Oral hypoglycemic activity of some medicinal plants of Sri Lanka. J Ethnopharmacol 11 : 223-231.

3) Platel K, Srinivasan K. 1997. Plant foods in the management of diabetes mellitus: vegetables as potential hypoglycemic agents. Nahrung 41: 68-74.

4) Leatherdale BA, Panesar RK, Sing G, Atkins TW, Bailey CJ, Bignell AHC. 1981. Improvement in glucose tolerance due to Momordica charantia (karela). $\mathrm{Br}$ Med J 282: 1823-1824.

5) Welihinda J, Kaunanayake EH, Sheriff MHR, Jayasinghe KSA. 1986. Effect of Momordica charantia on the glucose tolerance in maturity onset diabetes. J Ethnopharmacol 17: 277-282.

6) Sing N, Tyagi SD, Agarwal SC. 1989. Effects of long term feeding of acetone extract of Momordica charantia (whole fruit powder) on alloxan diabetic albino rats. Ind J Physiol Pharmacol 33: 97-100.

7) Akhtar MS, Athar MA, Yaqub M. 1981. Effect of Momordica charantia on blood glucose level of normal and alloxan-diabetic rats. Planta Med 42: 205-212.

8) Grover JK, Vats V, Rathi SS, Dawar R. 2001. Traditional Indian anti-diabetic plants attenuate progression of renal damage in streptozotocin induced diabetic mice. $J$ Ethnopharmacol 76: 233-238.

9) Bailey CJ, Day C, Turner SL, Leatherdale BA. 1985. Cerasee, a traditional treatment for diabetes. Studies in normal and streptozotocin diabetic mice. Diabetes Res 2: 81-84.

10) Jayasooriya AP, Sakono M, Yukizaki C, Kawano M, Yamamoto K, Fukuda N. 2000. Effects of Momordica charantia powder on serum glucose levels and various lipid parameters in rats fed with cholesterol-free and cholesterol-enriched diets. J Ethnopharmacol 72: $331-$ 336.

11) Senanayake GVK, Maruyama M, Shibuya K, Sakono M, Fukuda N, Morishita T, Yukizaki C, Kawano M, Ohta H. 2004. The effects of bitter melon (Momordica charantia) on serum and liver triglyceride levels in rats. J Ethno- pharmacol 91: 257-262.

12) Ahmed I, Lakhani MS, Gillett M, John A, Raza H. 2001. Hypotriglyceridemic and hypocholesterolemic effects of anti-diabetic Momordica charantia (karela) fruit extract in streptozotocin-induced diabetic rats. Diabetes Res Clin Pract 51: 155-161.

13) Chen Q, Chan LL, Li ET. 2003. Bitter melon (Momordica charantia) reduced adiposity, lowers serum insulin and normalizes glucose tolerance in rats fed a high fat diet. J Nutr 133: 1088-1093.

14) Adeli K, Taghibiglou C, Van Iderstine SC, Lewis GF. 2001. Mechanisms of hepatic very low-density lipoprotein overproduction in insulin resistance. Trends Cardiovasc Med 11: 170-176.

15) Ng TB, Wong CM, Li WW, Yeung HW. 1986. A steryl glycoside fraction from Momordica charantia seeds with an inhibitory action on lipid metabolism in vitro. Biochem Cell Biol 64: 766-771.

16) Griffiths KM, Bacic A, Howlett BJ. 2003. Sterol composition of mycelia of the plant pathogenic ascomycete Leptosphaeria maculans. Phytochemistry 62: 147-153.

17) American Institute of Nutrition. 1977. Report of the American Institute of Nutrition ad hoc committee on standards for nutritional studies. J Nutr 107: 13401348.

18) Folch J, Lees M, Sloane-Stanley GH. 1957. A simple method for the isolation and purification of total lipides from animal tissues. J Biol Chem 226: 497-509.

19) Fukuda N, Igari N, Etoh T, Hidaka T, Ikeda I, Sugano M. 1995. A comparison of the metabolism of cis, cis-, cis, trans/trans, cis- and trans/trans-9,12-octadecadienoic acids in rat liver. Nutr Res 13: 779-786.

20) Spurlock ME, Ji SQ, Godat RL, Kuske JL, Willis GM, Frank GR, Cornelius SG. 2001. Changes in the expression of uncoupling proteins and lipases in porcine adipose tissue and skeletal muscle during feed deprivation. J Nutr Biochem 12: 81-87.

21) Ontko JA, Cheng Q, Yamamoto M. 1990. Metabolic factors underlying high serum triglycerides in the normal hamster. J Lipid Res 31: 1983-1992.

22) Raman A, Lau C. 1996. Anti-diabetic properties and phytochemistry of Momordica charantia L. (Cucurbitaceae). Phytomedicine 2: 349-362.

23) Park KH, Shin HJ, Song YB, Hyun HC, Cho HJ, Ham HS, Yoo YB, Ko YC, Jun WT, Park HJ. 2002. Possible role of ginsenoside $\mathrm{Rb} 1$ on regulation of rat liver triglycerides. Biol Pharm Bull 25: 457-460. 